Enfermagem Brasil 2018;17(1):18-25

\title{
ARTIGO ORIGINAL \\ Perfil da população notificada por tuberculose em um hospital escola do interior paulista de 2010 a 2014
}

Mariana Bertolino Fioramonti dos Santos, M.Sc. ${ }^{*}$, Luciano Garcia Lourenção, D.Sc. ${ }^{* *}$, Margarete Artico Baptista, D.Sc. ${ }^{* * *}$, Cláudia Eli Gazetta, D.Sc. ${ }^{* * *}$

*Enfermeira Sênior do Instituto Israelita de Ensino e Pesquisa Albert Einstein, **Enfermeiro, Professor Titular-Livre da Escola de Enfermagem da Universidade Federal do Rio Grande (EEnf/FURG), ${ }^{* * \star}$ Enfermeira, Professora Adjunta do Departamento de Enfermagem em Saúde Coletiva e Orientação Profissional da Faculdade de Medicina de São José do Rio Preto (FAMERP), ${ }^{* * * * E n f e r m e i r a, ~ P r o f e s s o r a ~ A d j u n t a ~ d o ~ D e p a r t a m e n t o ~ d e ~ E n f e r m a g e m ~ e m ~ S a u ́ d e ~}$ Coletiva e Orientação Profissional da Faculdade de Medicina de São José do Rio Preto (FAMERP)

Recebido em 27 de setembro de 2017; aceito em 27 de outubro de 2017.

Endereço para correspondência: Mariana Bertolino Fioramonti dos Santos, Rua Santa Archelia, 185/405, Bloco 2, Jardim Casablanca, 05846-410 São Paulo SP, E-mail: mariana_br4@hotmail.com; Luciano Garcia Lourenção: luciano.famerp@gmail.com; Margarete Artico Baptista: marticobaptista@famerp.br; Cláudia Eli Gazetta: claudiagazetta@yahoo.com.br

\section{Resumo}

O estudo objetivou caracterizar a população notificada por tuberculose em um Hospital Escola referência do Estado de São Paulo. Estudo descritivo, retrospectivo, realizado em instituição hospitalar do interior do Estado de São Paulo, no período de janeiro de 2010 a dezembro de 2014. Os dados foram coletados a partir das fichas de notificação dos casos de tuberculose notificados pelo Núcleo de Vigilância Epidemiológica do serviço. Os dados mostraram que, nos aspectos sociodemográficos, a incidência de doença foi maior em homens $(73,5 \%)$ brancos $(70,7 \%)$, faixa etária entre 20 e 59 anos $(80,7 \%)$, com ensino fundamental incompleto $(48,5 \%)$, que trabalham em ocupações com baixos índices de remuneração e provenientes do próprio município $(57,6 \%)$. Em relação aos aspectos epidemiológicos, foi predominante caso novo (82,2\%), forma clínica pulmonar (61,8\%), apresentando coinfecção pelo HIV (36,7\%), sendo internado para elucidação diagnóstica $(46,0 \%)$, permanecendo no serviço por até 15 dias e recebendo alta hospitalar para tratamento ambulatorial (64,9\%). Os resultados confirmam a influência das condições socioeconômicas no adoecimento por tuberculose, na região estudada. Reforçam a existência de fragilidades nos serviços de atenção primária, que comprometem as ações de controle da tuberculose em nível local e regional, tornando o hospital uma importante porta de entrada para os doentes.

Palavras-chave: tuberculose, epidemiologia, incidência, hospitalização, notificação de doenças.

\section{Abstract \\ Profile of notified tuberculosis population in a school hospital of São Paulo from 2010 to 2014}

The study aimed to characterize the population notified by tuberculosis in a teaching hospital that is reference in the state of São Paulo. Descriptive and retrospective study, carried out in hospital in the State of São Paulo, from January 2010 to December 2014. Data were collected from the reporting forms of tuberculosis cases reported by the hospital's Surveillance Epidemiology Center service. The data shows that the sociodemographic aspects of the incidence of tuberculosis was highest in white (70.7\%) men (73.5\%), aged between 20 and 59 years $(80.7 \%)$, with incomplete primary education (48.5\%), working at occupations with low rates of payment and from the city itself $(57.6 \%)$. Regarding epidemiological aspects was predominantly new cases $(82.2 \%)$ with pulmonary clinical form $(61.8 \%)$, with co-infection with HIV $(36.7 \%)$ and the population was hospitalized for diagnostic elucidation $(46.0 \%)$, remaining in service for up to 15 days and receiving hospital for outpatient treatment $(64.9 \%)$. The results confirm the influence of socioeconomic conditions on the illness by tuberculosis in region studied, reinforce the existence of weaknesses in primary care services, which compromise the 
tuberculosis control actions at regional and local level, making the hospital a major gateway to the patients.

Key-words: tuberculosis, epidemiology, incidence, hospitalization, disease notification.

\section{Resumen \\ Perfil de la población notificada por tuberculosis en un hospital escuela del interior paulista de 2010 a 2014}

El estudio pretende caracterizar la población notificada de tuberculosis en un Hospital Escuela referencia del estado de São Paulo en Brasil. Estudio descriptivo, retrospectivo, realizado en hospital en el estado de São Paulo, desde enero de 2010 a diciembre de 2014. Se recolectaron los datos de la notificación de casos de tuberculosis notificados por el sistema de vigilancia epidemiológica del hospital. Los datos mostraron que, en los aspectos socio-demográficos, la incidencia de tuberculosis es mayor en hombres (73,5\%) blancos $(70.7 \%)$, edad entre 20 y 59 años $(80.7 \%)$, primaria incompleta $(48,5 \%)$, que trabajan en ocupaciones con bajos niveles de remuneración y provenientes del propio municipio (57.6\%). En lo referente a aspectos epidemiológicos fue predominante caso nuevo (82.2\%), con forma clínica pulmonar (61.8\%), mostrando la coinfección por VIH (36.7\%), siendo hospitalizados para la aclaración diagnóstica $(46,0 \%)$, permaneciendo un promedio de 15 días y recibiendo alta hospitalaria para tratamiento ambulatorio (64,9\%). Los resultados confirman la influencia de las condiciones socioeconómicas sobre la incidencia de enfermar por tuberculosis en la región estudiada. Refuerzan la fragilidad de los servicios de atención primaria, que comprometen las acciones del control de la tuberculosis en nivel local y regional, haciendo el hospital una importante puerta de entrada para los enfermos.

Palabras-clave: tuberculosis, epidemiología, incidencia, hospitalización, notificación de enfermedades.

Introdução

A tuberculose (TB) é uma doença infecciosa de evolução crônica, que compromete principalmente os pulmões, sendo o Mycobacterium tuberculosis 0 agente etiológico, descoberto por Robert Koch, em 1882. A transmissão da doença ocorre principalmente por via aérea e é facilitada pela aglomeração humana [1].

A TB se mantém no topo da lista dos agravos de saúde tanto no âmbito mundial, quanto no nacional. Segundo a Organização Mundial da Saúde (OMS) estima-se que, em 2014, havia 9,6 milhões de pessoas doentes por tuberculose no mundo e, destas, 1,5 milhão acabaram morrendo em decorrência da doença [1].

Segundo dados do Ministério da Saúde, o Brasil permanece na 16ำ posição, entre os 22 países que concentram $80 \%$ dos casos de tuberculose no mundo [2-3].

Em 2014 foram diagnosticados, no Brasil, 67.966 casos novos de tuberculose, com uma distribuição heterogênea entre as Unidades da Federação. O coeficiente de incidência da doença variou de 11 casos por 100 mil habitantes no estado de Goiás a 68,4 casos por 100 mil habitantes no estado de Amazonas e uma incidência média de 33,5 casos por 100 mil habitantes, abaixo da registrada em 2005 , no início da série histórica, quando se verificou uma taxa de incidência anual média de 41,5 casos por 100 mil habitantes [2-3].

A região Sudeste é a segunda região brasileira em incidência da doença. Com valores em torno de 36,2 casos por 100 mil habitantes, fica atrás apenas da região Norte [2-3]. O estado de São Paulo apresenta incidência maior, tanto em relação à média nacional quanto à regional, com taxa de 36,9 casos por 100 mil habitantes e distribuição heterogênea entre seus municípios [2].

Além da localização geográfica, outros aspectos como faixa etária, sexo, raça branca, baixa escolaridade e condições socioeconômicas são registrados e contabilizados, perfazendose assim a caracterização do grupo populacional mais susceptível a contrair a doença $[2,5,6]$. Segundo dados estaduais, em torno de $66 \%$ dos casos registrados em São Paulo ocorrem em homens e aproximadamente $70 \%$ das pessoas infectadas estão na faixa etária de 20 a 59 anos, além da importante incidência da doença sobre populações de poder econômico e social mais baixos $[2,5,6]$.

Atualmente uma pessoa infectada e sem tratamento pode contaminar, anualmente, entre dez e quinze pessoas, disseminando a infecção pela comunidade. Embora o diagnóstico possa ser realizado precocemente, a partir da busca ativa de sintomáticos respiratórios, pelas equipes da Atenção Básica, somente $60 \%$ dos casos são diagnosticados desta forma. Grande 
número de pacientes é diagnosticado apenas na rede hospitalar, que deveria ser utilizada em situações graves da doença, nos casos de pacientes que vivem em más condições socioeconômicas e/ou quando a doença se associa a outros agravos [7].

Quando inseridos nas instituições de saúde, os pacientes em tratamento hospitalar merecem atenção especial, pois apresentam elevado potencial de disseminação do bacilo intra-hospitalar, o que exige medidas de controle institucionais, desde a admissão até a alta hospitalar [8].

Ante o exposto, este estudo objetivou descrever as características sociodemográficas e epidemiológicas de pacientes com tuberculose, atendidos em um hospital escola do interior paulista, no período de 2010 a 2014.

\section{Material e métodos}

Estudo descritivo, retrospectivo, realizado em um Hospital Escola referenciado, na cidade de São José do Rio Preto, São Paulo, no período de janeiro de 2010 a dezembro de 2014.

O município está classificado no Grupo Um do Estado de São Paulo (Grupo de Elite), com bons níveis de riqueza, longevidade e escolaridade, de acordo com o Índice Paulista de Responsabilidade Social (IPRS) [9].

A rede hospitalar compreende oito hospitais, dentre os quais se destaca o Hospital de Base, uma fundação filantrópica com 720 leitos, que atende majoritariamente usuários do Sistema Único de Saúde (85\%). Em associação à Faculdade de Medicina de São José do Rio Preto (FAMERP) se tornou um centro de referência em saúde para uma região que compreende 102 municípios e mais de dois milhões de habitantes [9-10].

O Hospital de Base faz em média 12 mil atendimentos mensais de emergência e 30 mil atendimentos ambulatoriais, dentre os quais estão os atendimentos a pacientes com diagnóstico de tuberculose [10].

A coleta de dados foi realizada por meio da Ficha de Notificação/Investigação de Tuberculose [11], dos casos notificados pelo Núcleo Hospitalar de Epidemiologia (NHE) da instituição hospitalar. $\mathrm{O}$ instrumento contém dados que possibilitaram a caracterização social e demográfica, bem como as características da internação e as características epidemiológicas da população estudada.

A população estudada foi composta por 550 casos notificados por tuberculose no Hospital de Base de São José do Rio Preto, no período de primeiro de janeiro de 2010 a 31 de dezembro de 2014. Esta população foi submetida ao processo de pareamento de registros seguido de depuração de registros repetidos.

Os dados foram armazenados em planilhas eletrônicas e receberam o tratamento estatístico pelo programa Microsoft Excel®, através destes dados foram construídas tabelas e gráficos de frequência.

O projeto foi aprovado pelo Comitê de Ética em Pesquisa da Faculdade de Medicina de São José do Rio Preto, sob Parecer n. 300.788, de 11/06/2013.

Conforme mostra a Tabela I, em relação às características sociodemográficas da população estudada, houve predomínio do sexo masculino (73,5\%), na razão de 2,76 homens para uma mulher; faixa etária entre 20 e 59 anos (80,7\%); raça/cor branca $(70,7 \%)$; ensino fundamental incompleto (48,5\%); residente no município de São José do Rio Preto $(57,6 \%)$. Em relação à ocupação, destaca-se o número de pacientes em atividades do lar (16,2\%), da construção civil $(10,7 \%)$ e aposentados $(8,7 \%)$.

Conforme mostra a Tabela II, houve predomínio de casos novos como forma de ingresso $(82,2 \%)$; a forma clínica de tuberculose predominante foi a pulmonar $(61,8 \%)$ e HIV como agravo associado mais comum $(36,7 \%)$. O motivo da internação mais frequente foi elucidação diagnóstica $(46,0 \%)$ e o da ata hospitalar, tratamento ambulatorial $(64,9 \%)$. 
Tabela I - Características sociodemográficas dos casos de TB notificados pelo NHE do hospital do estudo no período de 2010-2014.

\begin{tabular}{lcc}
\multicolumn{1}{c}{ Variáveis } & $\mathbf{n}$ & $\%$ \\
\hline Sexo & & \\
Masculino & 404 & 73,5 \\
Feminino & 146 & 26,5 \\
Faixa etária & & \\
$0-19$ & 26 & 4,7 \\
$20-39$ & 208 & 37,8 \\
$40-59$ & 236 & 42,9 \\
60 e mais & 80 & 14,5 \\
Raça/Cor & & \\
Branca & 389 & 70,7 \\
Negra & 94 & 17,1 \\
Parda & 33 & 6,0 \\
Amarela & 3 & 0,5 \\
Ignorado & 31 & 5,6 \\
Escolaridade & & \\
Analfabeto & 15 & 2,7 \\
Ensino Fundamental Incompleto & 267 & 48,5 \\
Ensino Fundamental Completo & 61 & 11,1 \\
Ensino Médio Incompleto & 12 & 2,2 \\
Ensino Médico Completo & 68 & 12,4 \\
Ensino Superior Incompleto & 13 & 2,4 \\
Ensino Superior Completo & 13 & 2,4 \\
Ignorado & 101 & 18,4 \\
Município de Residência & & \\
São José do Rio Preto & 317 & 57,6 \\
Municípios da DRS XV & 212 & 38,5 \\
Demais municípios & 21 & 3,8 \\
Ocupação & & \\
Do lar & 89 & 16,2 \\
Construção Civil & 59 & 10,7 \\
Aposentado & 48 & 8,7 \\
Trabalhador Rural & 30 & 5,5 \\
Outros & 283 & 51,5 \\
Ignorado & 4,5 \\
\hline
\end{tabular}

Na Tabela III são apresentadas as características sobre a internação dos casos de TB notificados pelo hospital durante o período do estudo. Verificou-se que $518(94,2 \%)$ casos necessitaram internação. A maioria dos pacientes permaneceu internada por um período de 0 a 10 dias para tratamento $(43,4 \%)$. 
Tabela II - Características epidemiológicas dos casos de TB notificados pelo NHE do hospital do estudo no período de 2010-2014.

\begin{tabular}{lcc}
\multicolumn{1}{c}{ Variável } & $\mathbf{n}$ & $\%$ \\
\hline Forma de ingresso & 452 & 82,2 \\
Caso Novo & 41 & 7,5 \\
Reingresso & 48 & 8,7 \\
Recidiva & 9 & 1,6 \\
Outros & & \\
Forma clínica & 340 & 61,8 \\
Pulmonar & 42 & 7,6 \\
Meningoencefálica & 27 & 4,9 \\
Ganglionar periférica & 36 & 6,5 \\
Pleural & 16 & 2,9 \\
Miliar & 89 & 16,2 \\
Outros & & \\
Agravos associados & 202 & 36,7 \\
HIV & 183 & 33,3 \\
Tabagismo & 176 & 32,0 \\
Alcoolismo & 119 & 21,6 \\
Drogadição & & \\
Motivo da internação & & 46,0 \\
Elucidação diagnóstica & 253 & 7,3 \\
Hemoptise & 40 & 36,7 \\
Outros & 202 & 10,0 \\
Ignorado & 55 & \\
Motivo da alta hospitalar & & 64,9 \\
Tratamento ambulatorial & 357 & 6,4 \\
Óbito por TB & 35 & 7,1 \\
Óbito por outra causa & 39 & 5,3 \\
Outros & 29 & 16,4 \\
Sem informação & 90 & \\
\hline
\end{tabular}

Tabela III - Características da internação dos casos de TB notificados pelo NHE do hospital do estudo no período de 2010-2014.

\begin{tabular}{lcc}
\multicolumn{1}{c}{ Variável } & $\mathbf{n}$ & $\%$ \\
\hline Internação & 518 & 94,2 \\
Sim & 32 & 5,8 \\
Não & & \\
Período de internação & & 18,3 \\
0 a 5 dias & 95 & 25,1 \\
6 a 10 dias & 130 & 15,3 \\
11 a 15 dias & 79 & 10,8 \\
16 a 20 dias & 56 & 7,7 \\
21 a 25 dias & 40 & 5,4 \\
26 a 30 dias & 28 & 16,2 \\
31 dias ou mais & 84 & 1,2 \\
Sem informação & 6 & \\
\hline
\end{tabular}

\section{Discussão}

O estudo mostrou que, nos aspectos sociodemográficos, a incidência de TB é maior em homens brancos, na faixa etária entre 20 e 60 anos, com ensino fundamental incompleto, que trabalham em ocupações com baixos índices de remuneração e provenientes do próprio município. Nos aspectos epidemiológicos houve predomínio de casos novos, forma clínica pulmonar e com coinfecção pelo HIV, internação para elucidação diagnóstica e permanência no serviço por até 15 dias, com alta hospitalar para tratamento ambulatorial.

A predominância de doentes do sexo masculino corrobora o perfil observado em estudos não controlados sobre TB [11-15]. O predomínio da raça/cor branca condiz com o 
Censo Demográfico de 2010, que aponta população do estado de São Paulo composta, majoritariamente, por pessoas de cor ou raça branca $(69,9 \%)$ [16]. Diferentemente, estudo realizado no Maranhão, onde a população é predominantemente da raça/cor parda, apontou prevalência de doentes da raça/cor parda [17]. Estes dados demonstram que a raça/cor do dente de TB está intimamente ligada ao perfil demográfico da população, podendo variar entre as diferentes regiões do Brasil [11-12,17-19].

Em relação à idade dos doentes, a prevalência de adultos corresponde à faixa etária economicamente ativa da população, podendo acarretar impacto social importante, devido à inserção destas pessoas no mercado de trabalho [12-14]. A ocorrência dos casos de TB na população acima de 60 anos está relacionada, por um lado, ao aumento da expectativa de vida da população brasileira e, por outro, à susceptibilidade biológica do idoso, que aumenta o risco de infecção pelo bacilo. Há, ainda, a precariedade das condições de vida e acesso aos serviços de saúde, decorrentes da baixa renda familiar, dos déficits nutricionais e de cuidados com a saúde, que levam à reativação do bacilo, além das dificuldades de diagnóstico da doença nesta faixa etária [13-15].

Este estudo mostrou, também, um percentual significativo de doentes em ocupações que não exigem alta escolaridade, como trabalho rural, construção civil e do lar, reforçando a prevalência da ocorrência da tuberculose em populações com pouco estudo, baixo nível socioeconômico, condições laborais mais insalubres e com déficit de acesso aos serviços e cuidados de saúde [18-21]. A baixa escolaridade que se reflete nos segmentos sociais mais afetados do país aumenta a dificuldade de acesso à informação sobre medidas preventivas, diagnósticas e curativas da TB [12,18-19].

O ingresso hospitalar dos doentes como casos novos é corroborado por estudos realizados em outros hospitais brasileiros [13,17,19]. Esta característica da admissão hospitalar associada a taxas de recidiva e reingresso mais baixas favorece as chances de cura, no entanto, representam um dado alarmante, pois mostram um importante percentual da população que, por algum motivo, não têm acesso ao diagnóstico e tratamento adequados nos serviços de atenção primária $[21,22]$.

O predomínio da forma clínica pulmonar, observado neste estudo, confirma os resultados de estudos recentes. Nos casos extrapulmonares, chama atenção a forma meningoencefálica que, diferentemente de outros estudos, é a que mais acometeu nos sujeitos desta pesquisa, enquanto as formas ganglionar periférica e pleural tiveram menor ocorrência $[12-14,17,19]$.

As taxas de coinfecção TB/HIV observadas neste estudo foram superiores às observadas no distrito paulista de Capão Redondo [14], porém é condizente com estudos que relatam alta correlação entre fatores de risco para a contaminação pelo HIV e desenvolvimento de tuberculose $[12,17]$. Além disso, o índice de coinfecção TB/HIV observado foi superior ao encontrado em outro estudo realizado no município de São José do Rio Preto, em diferente época, indicando que a coinfecção vem aumentando ao longo dos anos na região estudada [12].

O percentual de óbitos por tuberculose observado no estudo foi relativamente baixo em relação a estudo realizado na cidade de São Paulo [23] e condizente com resultados observados em estudo desenvolvido em Niterói, Rio de Janeiro [24]. Por outro lado, se considerarmos a TB como causa secundária do óbito e somada à causa primária, o percentual observado é significativamente elevado e pode estar relacionado às fragilidades dos serviços de atenção primária do município e consequente demora no diagnóstico da doença [22].

O número de pacientes que necessitaram de internação para diagnóstico e tratamento da TB desperta, ainda, a discussão sobre a condição que estes indivíduos chegam ao serviço hospitalar, reforçando as fragilidades do primeiro atendimento recebido por este doente nos serviços de atenção primária e a responsabilidade dos profissionais no controle da TB. Apesar de estes resultados não condizerem com a hierarquização do sistema municipal de saúde, refletem a situação do diagnóstico do agravo no município e corroboram estudo anterior, que verificou que as instituições hospitalares são a principal porta de entrada do doente no município [22].

Logo, o diagnóstico hospitalar da TB, tanto de moradores do município de São José do Rio Preto, quanto dos outros 101 municípios de referência do Departamento Regional de Saúde (DRS XV), reforça a necessidade e importância da discussão sobre a eficiência dos programas de controle e tratamento da doença no nível de atenção primária destes municípios $[21,23]$. 
O período de internação observado foi, no entanto, menor que o relatado em estudos realizados em Rio de Janeiro [24] e Porto Alegre [25], cujo período de hospitalização foi superior a 15 dias. Estes dados podem estar relacionados à agilidade diagnóstica e eficácia do tratamento hospitalar.

Por fim, destaca-se o elevado percentual de registros ignorados ou sem informação, observados no estudo. Sabe-se que os estudos epidemiológicos da TB dependem de dados provenientes de sistemas de informação em saúde, coordenados pelo Ministério da Saúde e alimentados pelas unidades notificadoras e que, ao longo dos anos, esforços são realizados para melhorar estes sistemas de comunicação. Como essas informações servem de subsídio para a tomada de decisões gerenciais nos níveis nacional, estadual, regional e local, é fundamental que profissionais de saúde sejam capacitados e estejam sempre atentos às ações de busca ativa e notificação adequada dos casos, buscando maior resolutividade e efetividade no controle da doença $[26,27]$.

Apesar das limitações encontradas, inerentes aos estudos pautados em dados secundários (subnotificação dos casos, incompletude ou ilegibilidade dos dados, diferentes formas de preenchimento das fichas de notificação), os resultados evidenciaram o panorama geral da população atendida e notificada para tuberculose no hospital do estudo, referência para os 102 municípios integrantes do Departamento Regional de Saúde.

Os resultados obtidos mostram que a população acometida por TB é composta majoritariamente por homens brancos, adultos, em faixa etária economicamente ativa, baixa escolaridade, que trabalham em ocupações com baixos índices de remuneração e provenientes do próprio município, confirmando a influência das condições socioeconômicas no adoecimento.

Em relação aos aspectos epidemiológicos, a TB é notificada predominantemente como caso novo, na forma clínica pulmonar, com presença de coinfecção pelo HIV; internação para elucidação diagnóstica, com permanência média de 18 dias no serviço e alta hospitalar para tratamento ambulatorial.

Estes resultados confirmam a influência das condições socioeconômicas no adoecimento por TB, na região estudada. O estudo reforça a existência de fragilidades nos serviços de atenção primária, que comprometem as ações de controle da TB em nível local e regional, e fazem do hospital uma importante porta de entrada para os doentes.

Novos estudos, com abordagem qualitativa, podem contribuir para elucidar as causas de internação hospitalar, contribuindo para o aprimoramento de ações de diagnóstico e controle da TB na atenção primária, e redução dos atendimentos hospitalares.

1. World Health Organization [WHO]. Global tuberculosis report 2016. [citado 2017 Out 19]. Disponível em URL:

http://apps.who.int/iris/bitstream/10665/250441/1/9789241565394-eng.pdf?ua=1

2. Ministério da Saúde. Secretaria de Vigilância em Saúde. O controle da tuberculose no Brasil: avanços, inovações e desafios. Bol Epidemiol 2014;45(2):13.

3. Ministério da Saúde. Secretaria de Vigilância em Saúde. Tuberculose: alinhada com o social, afinada com a tecnologia. Bol Epidemiol 2013;44(2):6p.

4. Organização Mundial da Saúde. Global Tuberculosis Report 2014. [citado 2017 Out 20]. Disponível em: URL:

http://apps.who.int/iris/bitstream/10665/137094/1/9789241564809_eng.pdf?ua=1

5. Ministério da Saúde. Boletim epidemiológico da tuberculose 2012. Brasília; 2012. [citado 2017 Out 20]. Disponível em: URL:

http://portalsaude.saude.gov.br/images/pdf/2014/julho/23/BE-2012-43-Mar--o--Especial-Tuberculose.pdf.

6. Barros PG, Pinto ML, Silva TC, Silva EL, Figueiredo TMRM. Perfil epidemiológico dos casos de tuberculose extrapulmonar em um município do estado da Paraíba, 20012010. Cad Saúde Coletiva 2014;22(4):343-50.

7. Belo MTCT, Luiz RR, Hanson C, Selig L, Teixeira EG, Chalfoun T, et al. Tuberculose e gênero em um município prioritário no estado do Rio de Janeiro. J Bras Pneumol 2010;36(5):621-5. 
8. Almeida CPB, Skupien EC, Silva DR. Health care seeking behavior and patient delay in tuberculosis diagnosis. Cad Saúde Pública 2015;31(2):321-30.

9. Prefeitura de São José do Rio Preto. Secretaria Municipal de Planejamento Estratégico. Conjuntura Econômica 2015. 30 ed. São José do Rio Preto; 2015. [citado 2016 Jan 18]. Disponível em URL: http://www.riopreto.sp.gov.br/PortalGOV/do/subportais_Show?c=60202.

10. Hospital de Base de São José do Rio Preto. Abrangência [2017]. [citado 2017 Out 19]. Disponível em: URL: http://www.hospitaldebase.com.br/abrangencia.

11. Brasil, Ministério da Saúde, Sistema de Informação de Agravos de Notificação [SINAN]. Ficha de Notificação / Investigação Tuberculose 2014. [citado 2017 Out 19]. Disponível em URL:

http://portalsinan.saude.gov.br/images/documentos/Agravos/Tuberculose/Tuberculose_ v5.pd.

12. Santos MLSG, Ponce MAZ, Vendramini SHF, Villa TCS, Santos NSGM, Wysocki AD et al. A dimensão epidemiológica da coinfecção TB/HIV. Rev Latinoam Enferm 2009;17(5):683-8.

13. Gonçalves BD, Cavalini LT, Valente JG. Monitoramento epidemiológico da tuberculose em um hospital geral universitário. J Bras Pneumol 2010;36(3):347-55.

14. Hino P, Takahashi RF, Bertolozzi MR, Egry EY. Coinfecção de Tb/HIV em um distrito administrativo do município de São Paulo. Acta Paul Enferm 2012;25(5):755-61.

15. Oliveira AAV, Sá LD, Nogueira JA, Andrade SLE, Palha PF, Villa TCS. Diagnóstico da tuberculose em pessoas idosas: barreiras de acesso relacionadas aos serviços de saúde. Rev Esc Enferm USP 2013;47(1):145-51.

16. Instituto Brasileiro de Geografia e Estatística - IBGE. Censo 2010. [citado 2017 Out 26]. Disponível em: URL: https://censo2010.ibge.gov.br/resultados.html.

17. Santos Neto M, Silva FL, Sousa KR, Yamamura M, Popolin MP, Arcêncio RA. Perfil clínico e epidemiológico e prevalência da coinfecção tuberculose/HIV em uma regional de saúde no Maranhão. J Bras Pneumol 2012;38(6):724-32.

18. Prado TN, Caus AL, Marques M, Maciel EL, Golub JE, Miranda AE. Perfil epidemiológico de pacientes adultos com tuberculose e AIDS no estado do Espírito Santo, Brasil: relacionamento dos bancos de dados de tuberculose e AIDS. J Bras Pneumol 2011;37(1):93-9.

19. Cavalcante EFO, Silva DMGV. Perfil de pessoas acometidas por Tuberculose. Rev Rene 2013;14(4):720-9.

20. Basta PC, Marques M, Oliveira RL, Cunha EAT, Resendes APC, Souza-Santos R. Desigualdades sociais e tuberculose: análise segundo raça/cor, Mato Grosso do Sul. Rev Saúde Pública 2013;47(5):854-64.

21. Sassaki CM, Scatena LM, Gonzales RIC, Ruffino-Netto A, Hino P, Villa TCS. Predictors of favorable results in pulmonary tuberculosis treatment (Recife, Pernambuco, Brazil, 2001-2004). Rev Esc Enferm USP 2010;44(2):504-10.

22. Ponce MAZ, Wysocki AD, Scatolin BE, Andrade RLP, Arakawa T, Ruffino Netto $A$ et al. Diagnóstico da tuberculose: desempenho do primeiro serviço de saúde procurado em São José do Rio Preto, São Paulo, Brasil. Cad Saúde Pública 2013;29(5):945-54.

23. Perrechi MCT, Ribeiro SA. Desfechos de tratamento de tuberculose em pacientes hospitalizados e não hospitalizados no município de São Paulo. J Bras Pneumol 2011;37(6):783-90.

24. Orofino RL, Brasil PEA, Trajman A, Schmaltz CAS, Dalcolmo M, Rolla VC. Preditores dos desfechos do tratamento da tuberculose. J Bras Pneumol 2012;38(1):88-97.

25. Silva DR, Silva LP, Dalcin PTR. Tuberculose em pacientes hospitalizados: características clínicas dos pacientes que iniciaram tratamento nas primeiras $24 \mathrm{~h}$ de permanência hospitalar. J Bras Pneumol 2014;40(3):279-85.

26. Barreto ML, Teixeira MG, Bastos FI, Ximenes RAA, Barata RB, Rodrigues LC. Successes and failures in the control of infectious diseases in Brazil: social and environmental context, policies, interventions, and research needs. The Lancet 2011;377(9780):1877-89.

27. Santos TMMG, Nogueira LT, Arcêncio RA. Atuação de profissionais da Estratégia Saúde da Família no Controle da Tuberculose. Acta Paul Enferm 2012;25(6):954-61. 